\title{
The Stereo Communication Method of Artistic Visual Image Based on CAD Platform
}

\author{
Kai Gao $\mathbb{D}$ and Yong Wang \\ Department of Art Design, School of Education and Art, Shandong Institute of Petroleum and Chemical Technology, \\ Dongying City, Shandong Province 257061, China
}

Correspondence should be addressed to Kai Gao; 000420@slcupc.edu.cn

Received 25 October 2021; Revised 8 November 2021; Accepted 2 December 2021; Published 27 February 2022

Academic Editor: Bai Yuan Ding

Copyright (c) 2022 Kai Gao and Yong Wang. This is an open access article distributed under the Creative Commons Attribution License, which permits unrestricted use, distribution, and reproduction in any medium, provided the original work is properly cited.

\begin{abstract}
Due to the large amount of noise in the image background, the traditional methods have the problems of poor image communication effect, slow image communication efficiency, and poor noise processing effect. A three-dimensional communication method of art visual image based on CAD platform is proposed. Wavelet transform is used to extract artistic visual image features, and the fuzzy clustering method is used to standardize the obtained image feature data. Based on the data processing results, the CAD platform is constructed, the software program is designed, and the image chain coding is realized at the same time. The particle swarm optimization algorithm is used to convey the image of the obtained CAD general vectorization file, so as to complete the three-dimensional transmission of artistic visual image. The experimental results show that the image communication effect of this method is better, the image communication efficiency is higher, the noise suppression effect is better, the visual communication effect of the image is improved, and the performance of the designed method is superior.
\end{abstract}

\section{Introduction}

In recent years, the worldwide informatization process has been accelerating, and the development of all kinds of data has shown a soaring trend. As a key type of data in all kinds of information, image data can more intuitively record the state of human life. The subsequent technical problems of data analysis have gradually attracted people's attention, which has promoted the development of image visual communication research [1-3]. In the research of traditional image visual communication methods, a more scientific image extraction method is selected to obtain the image data, continuously track the data information, ensure the safety of the data in the acquisition process, and use the multifunctional algorithm to analyze the collected information, so as to enhance the reliability of the data information [4-6]. The data protection operation according to the corresponding data management rules improves the effect of image visual communication, but the processing effect of image features in the operation process is poor, and the image data information of the remaining part cannot be mastered. Therefore, it is necessary to study effective image visual communication methods to analyze and solve the above problems $[7,8]$.

In order to improve the effect of image visual communication, after a long time of research and development, some image communication methods have been formed. Reference [9] proposes an optimization method of plane visual communication effect based on wavelet change. The image is decomposed by wavelet and reconstructed by wavelet. In the process of reconstruction, the modulus diagram and phase angle diagram are calculated, and the edge images of each scale are extracted. Through the edge image, the corresponding edge points of the semireconstructed image are enhanced. On the basis of the above, the SLIP model is used to calculate the graphic beautification vector. In order to simplify the operation, the above calculation method is converted into a simple mathematical operation, and the image visual transmission effect is optimized through the reflected light graphic mode. The experimental results show that this method effectively optimizes the cost of image plane visual transmission, but some image details 
are not clear and the visual effect is not good. Reference [10] proposes a plane visual communication design method based on user experience effect. Computer vision imaging technology is used to sample the image information of plane visual communication, combined with the edge contour extraction method to detect the image contour of plane visual communication design, and extract the multiscale local structure feature information of the plane visual image. According to the needs of user experience effect, the boundary feature detection and multilevel structure decomposition in the process of plane visual communication design are carried out, the low-level visual structure of plane visual communication design is reconstructed by the method of adjacent pixel information fusion, and the user experience effect evaluation model of plane visual design image is established. According to the user experience effect, realize the optimization of plane visual communication design. The simulation test results show that this method has a good user experience effect and improves the design effect of plane visual communication, but the noise in the image background will affect the visual communication results. Reference [11] designed a visual communication system of animated character graphics and images in a virtual reality environment. In the hardware design, various adaptation parameters of the renderer motherboard were designed to optimize the experience of visual communication. In the software design, by introducing a Sobel edge operator, the gray function is established to solve the gradient amplitude, and the threshold is selected to compare it to complete the recognition and thinning of the edge data of animated character graphics and images. Design the motion capture module, establish the behavior control model, generate and manage the motion capture files, and complete the overall design of the system. The effectiveness of the system is verified by simulation experiments, but the efficiency of image visual communication is not high due to the large number of images.

Considering the large number of images and the noise in the image background, in order to improve the image communication effect, improve the image communication efficiency, and reduce the influence of noise factors on images, a three-dimensional communication method of artistic visual image based on the CAD platform is proposed.

\section{Art Visual Image Preprocessing}

There is a certain complementary relationship between the three-dimensional communication effect of an artistic visual image and color, among which the most obvious characteristics are reflected in the matching of color and the presentation of light feeling. The beauty of visual art is mainly reflected in its certain color openness, which is also the embodiment of a free concept in aesthetics and the theoretical basis of the three-dimensional communication method of artistic visual image.

\subsection{Feature Extraction of Art Visual Image Based on Wavelet} Transform. Visual art is a kind of humanities juxtaposed with architecture, music, literature, film, and other forms. It is an art form that can be perceived and understood by vision. It is a language means based on vision and guided by artistic visual images, such as paintings, photographic pictures, and film and television pictures. All visual artworks and images have highly important language as the intervention point. Artistic visual image is a visual cultural symbol that visually presents the law of art, artistic concept, artistic style, and characteristics of the times. Artistic visual images are full of deep cultural connotations. Image refers to the picture with visual effect. It is a way of information transmission different from words created by human beings through imitation or imagination of the natural world from the level of visual cognition and feeling. It is one of the most commonly used information carriers in human social activities. Artistic image is a kind of beautiful creation. It visually records the process of human civilization and is the main part of visual art. Its production and consumption are always the most basic communication activities in human social life and an invisible force to condense the society. Image chain coding is a binary image representation method based on the region boundary of binary image. This method can transform two-dimensional image into one-dimensional digital sequence. For large-scale artistic visual images, image chain coding can greatly save storage space and improve processing speed. The chain coding diagram in four directions is shown in Figure 1.

In Figure 1, the coding of using edges to calibrate the image area is image chain coding. Four chain codes 1, 2, 3, and 4 are used to represent the up, left, down, and right directions. Starting from any pixel on the image boundary, walk counterclockwise along the boundary pixel of the image and return to the starting point. The walking direction on the recording edge forms an ordered chain composed of direction chain code. With the starting point coordinates, the image boundary can be uniquely determined.

Wavelet transform is a local transform of time and frequency. It has the characteristics of multiresolution analysis and has the ability to characterize the local characteristics of signals in time domain and frequency domain. Wavelet transform can concentrate the energy of the original image on a small part of wavelet coefficients, and the decomposed wavelet coefficients have high local correlation in the detail components in three directions, which provides a powerful condition for feature extraction. Texture feature extraction using wavelet transform has been widely used in texture analysis, image compression, and surface defect detection of industrial products. Wavelet transform is often used for signal multiresolution decomposition [12], assuming the signal is $g(x)$; the discrete wavelet decomposition formula is

$$
g(x)==\sum_{i=1}^{n} e\left(x_{i}\right) \times \sum_{j=1}^{n} e\left(x_{j}\right),
$$

where $e\left(x_{i}\right)$ represents the scaling function, $e\left(x_{j}\right)$ represents the wavelet function, $i$ represents the scaling coefficient, and $j$ represents the wavelet coefficient.

As a two-dimensional function, the image is constructed by one two-dimensional scaling function $\mu(a, b)$ and three 


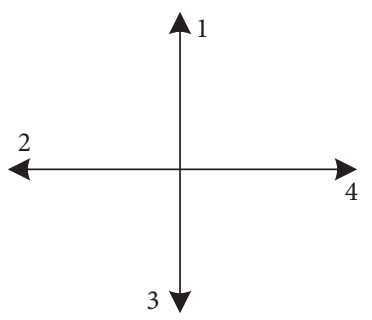

FIgURE 1: Chain coding diagram in four directions.

two-dimensional wavelet functions $\vartheta^{S}(a, b), \vartheta^{K}(a, b)$, and $\vartheta^{L}(a, b)$. The wavelet function measures the change of image gray in different directions: $\vartheta^{S}(a, b)$ measures the change of gray along the column direction, $\vartheta^{K}(a, b)$ measures the change of gray along the row direction, and $\vartheta^{L}(a, b)$ measures the change of gray along the diagonal direction [13]. Therefore, the discrete wavelet transform of the image $F(a, b)$ of size $m \times n$ is

$$
F(a, b)=\sum_{k=1}^{n} e(k)+\mu[e(k)+e(k-1)],
$$

where $e(k)$ represents the high-frequency coefficient and $e(k-1)$ represents the low-frequency coefficient.

According to formula (2), it can be seen that the wavelet transform decomposes the original image into a low-frequency part and a high-frequency part, and different levels of wavelet decomposition can obtain low-frequency images of different scales. After the $k$-level wavelet decomposition, reset the high-frequency part to 0 , and only keep the lowfrequency part, reconstruct the original image according to the modified high-frequency information and the unmodified low-frequency information, and obtain an approximate image $F^{\prime}(a, b)$ of the original image $F(a, b)$. This image contains the low-frequency information of the original image [14]. In the logarithmic domain, the difference between the original image and the approximate image is used to obtain the reflection component map $R(a, b)$ of the original image. Assuming that the original image undergoes $K$-level wavelet decomposition, $K_{a b}$ approximate images at different scales will be generated, and then, $R_{a b}$ reflection component maps will be obtained, combined with $R_{a b}$ reflection component maps to construct a visual image multiscale reflection model [15], that is,

$$
R_{a b}=\sqrt{\frac{\sum_{i=1}^{m} \sum_{j=1}^{n}\left[r_{i j}(x)-g(x)\right]^{2}}{\mathrm{~d} x}},
$$

where $r_{i j}(x)$ represents a nonlinear function, and this article chooses the arctangent function, because the arctangent function can reduce high-frequency noise to a certain extent.

The new media era is an image-centered information age. The mainstream consumption form of visual culture has been transformed into the production and consumption of artistic images based on digital and Internet technology. It can be said that digital and Internet technology is the technical basis of the new media era. It is leading to a revolution of visual culture, affecting people's production and consumption of artistic images at an unimaginable speed, thus imperceptibly changing people's inherent values and social ideology. While art image consumption has gradually become a popular way in this era, it is also pregnant with new art image forms and art language, and is tending to create art image culture that meets the needs of the new media era. In the new media era, the consumer of visual culture has gradually transformed into an imagecentered consumer behavior. Due to the convenience of digital and Internet technology for visual information transmission, it greatly improves its superiority for carrying image text, so as to transform the traditional consumption mode of visual culture into the consumption mode dominated by image.

In the high-frequency information obtained by wavelet decomposition of the original image, most of the information corresponds to the reflection components of the objects in the image scene, and the high-frequency information corresponding to the shadow part is relatively small. Therefore, the high-frequency information representing the shadow part can be regarded as the noise in the high-frequency information, and the noise can be detected by using the wavelet-based search method [16-18].

Let $U_{(p, v)}$ denote the wavelet coefficients obtained after the $p$-level wavelet decomposition of the artistic visual image, where $v=0,1,2,3$ corresponds to different subbands. For each pixel $(a, b)$ in the high-frequency subband, the threshold $T$ is used to determine whether it corresponds to the frequency of the shaded part.

$$
\sigma_{(p, v)}(a, b)= \begin{cases}1\left(U_{(p, v)}(a, b)\right)^{2}, & <T, \\ 0, & \text { others, }\end{cases}
$$

where $\sigma_{(p, v)}$ represents the detection result of whether the pixels in the subband $v$ represent shadows after the $p$-level decomposition of the artistic vision image. Among them, the calculation formula of the threshold $T$ is

$$
T=\frac{y_{i}^{u}-x_{j}^{u}}{r_{a}}
$$

where $y_{i}^{u}$ represents the calculation result of the difference in the brightness value of the pixel; $x_{j}^{u}$ represents the calculation result of the threshold value of the brightness of the pixel; and $r_{a}$ represents the shadow intensity index.

According to the updated wavelet coefficients, reconstruct the approximate image $F^{\prime}(a, b)$ of the original image $F(a, b)$, that is,

$$
F^{\prime}(a, b)=S^{-1} x^{v} x^{u}
$$

where $S^{-1}$ represents the inverse wavelet transform operator.

The final artistic visual image features can be extracted by the multiscale reflection model:

$$
S_{h}=\sum_{j=1}^{n} \partial\left[F(a, b)-F_{j}^{\prime}(a, b)\right] .
$$

The artistic visual image features are obtained by wavelet transform. These features are expressed as global features and local features. According to the artistic visual image 
content, the global features can be divided into color features, texture features, shape features, and spatial features. Local features are stable enough and have good discrimination in artistic visual images; Local features and local features are important aspects of art visual image feature acquisition, which provide parameter basis for further image processing.

\subsection{Data Processing of Artistic Vision Image Processing} Results. Before the artistic visual image is communicated, the acquired image feature data are standardized. Because the actual result data obtained are not only different in dimension meaning, but also in data variables. Therefore, in order to improve the validity and authenticity of the data processing results, the problem of excessive data difference is solved from a mathematical point of view.

Use fuzzy clustering method to transform the data; then, the data processing process is expressed by the following formula:

$$
G_{k}=\rho \varepsilon^{2}-\left(R_{\varepsilon}+S_{\varepsilon}\right),
$$

where $\varepsilon^{2}$ represents the sample data set matrix and $\rho$ represents the initial variable. Through the analysis of this formula, the clustering data processing formula can be obtained, as follows:

$$
V_{j}=\frac{\left[\sum_{i=1}^{n}\left(x_{i j}-\overline{x_{i j}}\right)^{2}\right]}{N^{2}},
$$

where $n$ represents the number of sample data processing times and $V_{j}$ represents the sample data collection after clustering $X_{i j}$. Expand this formula to obtain the data cluster center; the specific formula is as follows:

$$
X_{i j}=\frac{x_{i j}-\overline{x_{i j}}}{V_{j}} .
$$

Using the above formula, the standard sample data in $X_{i j}$ can be obtained, and the processed sample data matrix can be expressed as

$$
X=\left[\begin{array}{cccc}
x_{11} & x_{12} & \ldots & x_{1 n} \\
x_{21} & x_{22} & \ldots & x_{2 n} \\
\ldots & \ldots & \ldots & \ldots \\
x_{m 1} & x_{m 2} & \ldots & x_{m n}
\end{array}\right] .
$$

Through the above process, the basic data processing is completed. In the process of artistic visual image processing and analysis, the data are processed hierarchically through data statistics and dimensionality reduction. Due to the variety and amount of data, the original sample data are processed dimensionless. The general data processing methods include standardized processing and average processing. In the design of this method, MATLAB 6.5 statistical software package is used as the carrier of data processing to complete the high-precision data processing process.

\section{Stereo Communication Method of Artistic Visual Image Based on CAD Platform}

Based on the processed artistic visual image data, carry out the visual communication operation of artistic visual image, and use the computer and its graphics equipment to help the designers carry out the design work. Computer-aided design (CAD) is an effective means for the quality control of industrial parts design, manufacturing, and data processing. It emphasizes human-computer interaction design, operation setting, graphic display, and so on [19-21]. The CAD platform usually displays terminal graphics input version, drawing, scanning, printer, tape drive, and various software platforms with interactive calculation with graphics function. The overall structure of the CAD platform adopts a typical client/server network structure. The server and all clients are connected to the LAN, and then the collaborative scheme of distributed and centralized is adopted; that is, most functions are distributed on each site, such as interface programs, CAD software, and common information and program components. Only the site loading management module and shared information module of the initiator or organizer are designed, which are expressed as certain server functions. The client and server first establish communication with their respective CAD platforms through the CAD platform interface function and then establish a network connection based on TCP protocol, which is convenient for the collaborative design program of the client and server to form the connection between the three-dimensional CAD platforms at both ends, and establish an artistic visual image to convey the design information. Since CAD technology can provide a feature source for visual recognition, the CAD platform can be used to comprehensively process images. Therefore, the CAD platform method is used to obtain the three-dimensional communication strategy of artistic visual images to complete different visual tasks.

3.1. CAD Platform Construction. In the traditional sense, related platforms can only process $\mathrm{G}$ code files during the design process. If there is a CAD/CAM interface beforehand, CAD standard dwg/dxf files can be preprocessed and converted into $\mathrm{G}$ code input files, and then handed over to the system deal with. But when the system encounters handdrawn sketches, scanned images, engineering blueprints or digital photos, traditional systems lack effective recognition methods [22]. Therefore, this article focuses on the preprocessing technology and method of the CAD platform, including the database storage and extraction technology and the import and export graphics and image technology. The following is a specific discussion and analysis.

3.1.1. CAD Platform Software Program Design. In view of the defects and shortcomings of the previous CAD platform, a new CAD platform was further constructed and realized. It has some characteristics such as integration, interaction, popularization, networking, and parallelization. These are also the requirements of a new generation of software [23]. The CAD platform uses object-oriented technology, with 
Visual C ++6.0 as the development tool, SQL 2000 as the data library support, AutoCAD 2000 for the production of the library, and $\mathrm{C} / \mathrm{S}$ mode development. Figure 2 is a schematic diagram of the platform architecture.

According to Figure 2, the following is a detailed analysis of the database library storage and extraction technology and the import and export graphics and image technology of the designed CAD platform:

(1) Database storage and extraction technology: the CAD platform needs to call a large number of functional component symbols, browse, and call, using the client/server C/S (Client/Server) mode, and the graphic data module is stored on the server for remote computer access. Common database interfaces on Windows systems include ODBC (Open Database Interconnection), MFC (Microsoft Foundation Class), ODBC class, DAO (Data Access Object), RDO (Remote Data Object), OLEDB (Object Link Embedded Database), and ADO (ActiveX Data Object) [24]. ADO provides a database programming object model, simplifies OLEDB, belongs to the high-level database interface, and provides an automation interface. Therefore, use ADO mode to initialize OLE/COM library environment $\mathrm{A}$ fxO leInit (); the definition of ADO class is stored in ADO DLL (m sado15. $\mathrm{d} \mathrm{ll}$ ) as a resource, and then the ADO library file is imported with direct import symbol \#import in stdafx.h file to enable the compiler to compile correctly.

(2) Import and export graphics and image technology: each type of graphics software has its own storage format and file type. In order to share and interact with information between different systems, different file formats are required to be exchanged or converted. At present, the most commonly used data exchange method is star exchange; that is, each system uses standard data exchange format for indirect exchange [25]. With the help of the AutoCAD's DXF file structure, this article refers to the DXF file format: title section, table section, block section, element section, and file end, and establishes a CDXF class that can read and write the dxf file format. In addition, it also realizes image calling and processing.

3.1.2. Image Chain Coding Processing. After the art vision image is refined by the CAD platform, it is transformed into a single-pixel image, but it is still a binary image. To become a vectorized image, it needs to be coded and simplified by chain coding. The encoded image information can be conveniently used to extract the geometric features of the image to pave the way for the subsequent stereoscopic transmission of the image [26]. In addition, the chain-coded images can greatly save storage space and can store massive CAD data. The Freeman chain code method is a universal method widely used internationally to turn a single-pixel graphic into a chain code. The principle of Freeman chain

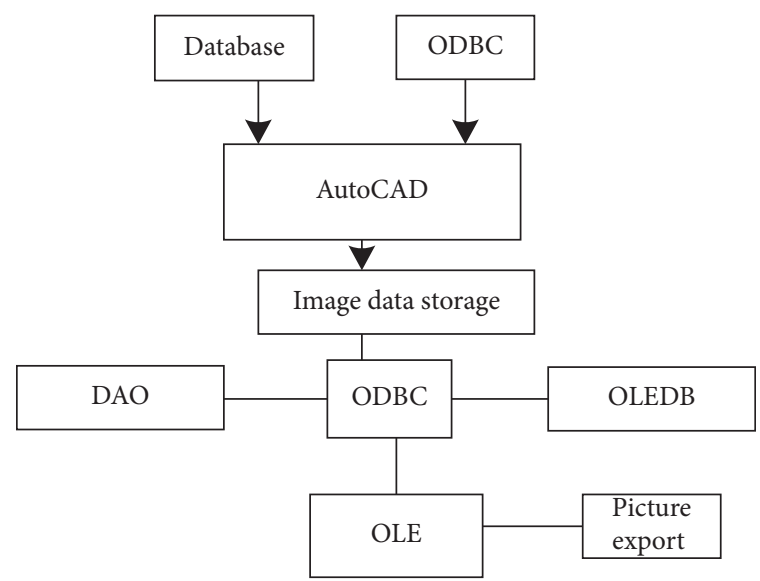

Figure 2: Schematic diagram of CAD platform structure.

code is to treat a binary image as a raster image composed of grid points. The method of chain coding is to express a continuous curve with the direction between adjacent pixels in the grid point theory. It can be divided into 4-domain chain codes and 8-domain chain codes. The chain coding process in this article adopts the 8-domain Freeman chain code method. Figure 3 is a schematic diagram of 8-domain chain codes.

Using the method from bottom to top, from left to right, scan the artistic vision image in turn, and the curves in the final image are represented as a two-dimensional array. The rows of the array indicate that there are several continuous curves in the image, and the columns of the array indicate the chain code trend of each curve. In the process of chain code scanning, the starting position of the curve must first be judged $[27,28]$. The pixel degree is calculated to determine, which pixel is the starting point of the chain code and which pixel is the ending point of the chain code. And mark the start point and end point, respectively, and record their coordinates. When scanning to the starting point, it will follow the direction of the starting point adjacent to the target pixel to the target end, and at the same time record the direction number of the current pixel relative to the previous pixel, and finally complete the chain coding of a curve $[29,30]$.

At this point, the CAD preprocessing process is set up, and the generated $\mathrm{dxf}$ file is the CAD universal vectorized file format, which can be imported into CAPP software for subsequent image stereo transmission processing.

\subsection{Realization of Stereo Communication of Artistic Vision} Images Based on Particle Swarm Optimization Algorithm. On the basis of using the CAD platform to obtain the CAD general vector file format, the image transmission method based on the particle swarm optimization algorithm is used to complete the three-dimensional transmission of artistic visual images [31]. To transform the art visual image stereo communication problem into the classification problem of image target foreground and image target background, the art visual image needs to be stereo transmitted under visual communication. The ratio function between the image 


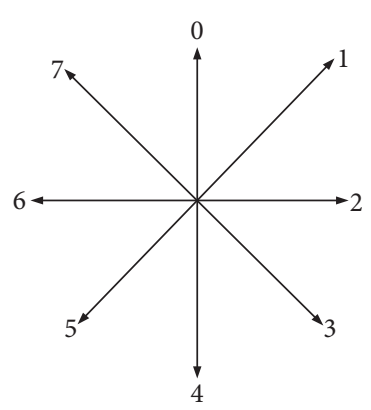

FIgURE 3: Original image.

feature distribution variance in the target foreground range is the separation coefficient to judge the foreground range of the image target, and the ratio function between the variance of image feature distribution in the target background range is the separation coefficient to judge the target background range of the image. In order to complete the three-dimensional communication of art visual image, particle swarm optimization algorithm is used to weight the feature components of art visual image and carry out real-time image target visual communication [32]. The process is as follows.

In order to understand the distinguishability of each range of artistic visual image, the corresponding log-likelihood ratio function variance formula is obtained on the premise of variance calculation:

$$
y(t)=e^{\omega} A \frac{z}{\sqrt{z^{2}+c^{2}}},
$$

where the log-likelihood ratio function is $e^{\omega}$, and the foreground range scale and the background range scale of the artistic visual image are $z^{2}$ and $c^{2}$, respectively. Use a vector to describe the weight $\varsigma$ :

$$
\varsigma=\left[\varsigma_{1}, \varsigma_{2}, \varsigma_{3}\right] \text {. }
$$

In the above formula, the feature vector of artistic visual image is $\varsigma_{1}, \varsigma_{2}, \varsigma_{3}$. Assuming that $\varsigma^{\prime}$ is the optimal solution of $\varsigma$, the particle swarm optimization algorithm is used to extract the optimal solution $\varsigma^{\prime}$ of different feature component weights of artistic vision images.

The steps of particle swarm optimization algorithm to extract $\varsigma^{\prime}$ are as follows.

Assuming that there are $\eta$ particles $\left\{\eta_{1}, \eta_{2}, \eta_{n}\right\}$ and $\varsigma$ in the same dimensional space, each particle $\eta_{i}$ has a solution for $\varsigma$, and the orientation of each particle in the group is determined by the fitness function value corresponding to the particle $[33,34]$.

Suppose the dimension of $\varsigma$ is $\mu$, the size of the particle swarm is $\sigma^{2}$, each particle in the swarm is 1 , and the orientation of each particle in the $t$ step iteration is

$$
\varsigma_{\mu}\left(\sigma^{2}\right)=\left(\varsigma_{\mu 1}, \varsigma_{\mu 2}, \ldots, \varsigma_{\mu i}, \ldots, \varsigma_{\mu N}\right)^{t} .
$$

The local optimal solution corresponding to the orientation of the best fitness of each particle is

$$
Q_{\mu}\left(\sigma^{2}\right)=\left(q_{\mu 1}, q_{\mu 2}, \ldots, q_{\mu i}, \ldots, q_{\mu N}\right) .
$$

The solution that reflects the best fitness of the particle in the local optimal solution is the current particle swarm optimal solution $Q_{k}$. The population adjusts the current collection speed and orientation of each particle through multiple iterations [35], and the iteration expression is

$$
\begin{aligned}
& Q_{k}=E_{e q} k_{s} \sqrt{K_{D}} \times \frac{f^{\beta} y^{\beta}}{V_{c}}+E_{e q} \sqrt{K_{D}} K_{0} \times \frac{f^{\gamma} y^{\gamma}}{V_{l}}, \\
& Q_{\mu}(t+1)=Q_{\mu}(t)+\varsigma_{\mu}(t+1) .
\end{aligned}
$$

In formulas (16) and (17), the learning factors are $f^{\beta}$ and $f^{\gamma}$, the uniform random numbers in the $[0,1]$ area are $y^{\beta}$ and $y^{\gamma}$, the collection speed of each particle in the $t$ step is $K_{D}$, the each particle collection speed in the $t+1$ is $K_{0}$, and the current azimuths of the population particles in steps $t$ and $t+1$ are $V_{c}$ and $V_{l}$, respectively.

The optimal solution $Q_{k}$ sought in this article is obtained after the iterative exploration of all particles in the population is completed, and there is no change in the orientation of each particle. It successfully realizes the tracking of the target feature of the artistic vision image and completes the stereoscopic vision transmission of the image target feature. Then, use the transmission value to sort the image data, which is easy to operate, reduces the complexity of image processing and completes the three-dimensional transmission of artistic visual images.

\section{Simulation Experiment Analysis}

In order to test the research effect of art visual image threedimensional communication method based on CAD platform, the simulation experiment needs to be carried out on the CAD platform, and the experimental results are compared in the form of pictures. In order to build a CAD platform that met the experimental conditions, the development script language of $3 \mathrm{D}$ core engine is java language. The editor tool can realize the high integration of various functions of the CAD platform, fuse and match the virtual scene in the artistic visual image with the task graphic image, and use the editor tool to reduce the operation complexity of the experiment. The 3D player loads the system content in the artistic visual image in a component-based way. Through the $3 \mathrm{D}$ player, you can experience the virtual reality effect, which provides an effective way for the display of artistic visual image effect. After the construction of the experimental platform, the simulation experiment is carried out on the platform. The research effect is compared with the traditional plane visual communication effect optimization method based on wavelet change and the plane visual communication design method based on user experience effect, and the experimental results are analyzed.

4.1. Experimental Data Set. The images used in this experiment are from CIFAR open-source database [36]. CIFAR is collected and sorted by Alex Krizhevsky, Vinod Nair, and Geoffrey Hinton; 60000 of the 800000 images in visual dictionary are selected and divided into CIFAR-10 and CIFAR-100. CIFAR-10 dataset contains $6000032 * 32$ color 


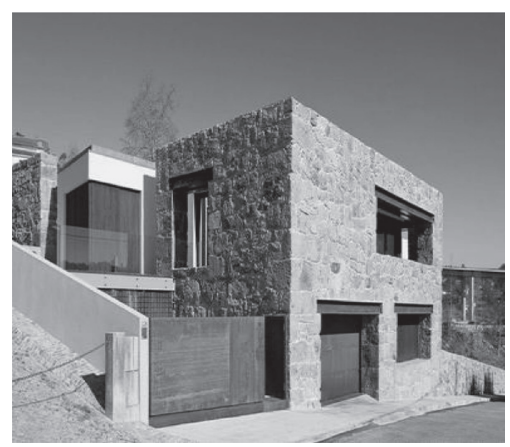

Original image

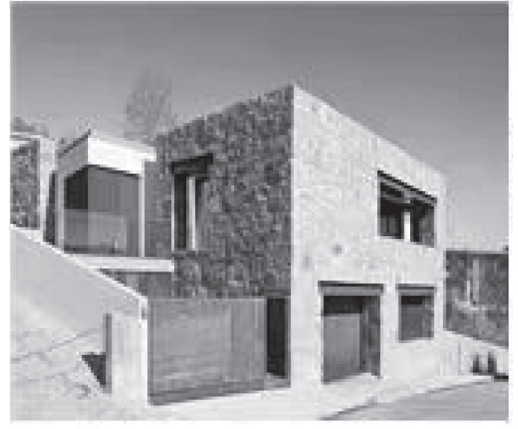

(a)

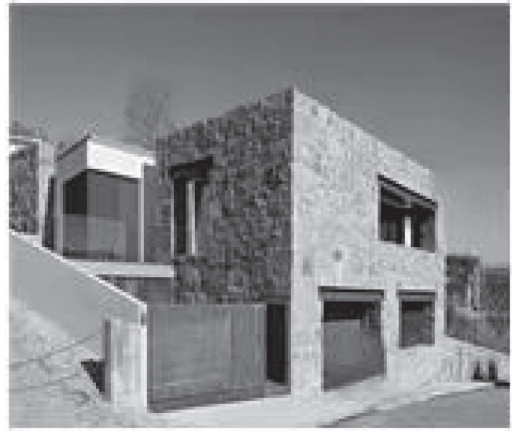

(a)

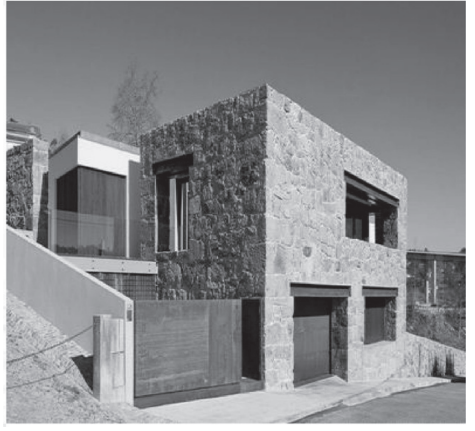

(a)

FIgURE 4: Comparison of image visual communication effects.

images, with 10 categories in total. There are 50000 training images and 10000 test images. The dataset is divided into five training blocks and one test block, and each block has 10000 images. The test block contains 1000 images randomly selected from each class, the training block contains these images in random order, and the training block contains 5000 images per class. The CIFAR-100 dataset contains 100 subclasses, each of which contains 600 images, including 500 training images and 100 test images. 100 categories are divided into 20 categories. Different types of artistic visual images are randomly selected from the database. In order to ensure the consistency of experimental conditions, 680 pixels are ensured $\times 480$ pixels is the size of each film and television image.

\subsection{Analysis of Experimental Results}

4.2.1. Image Visual Transmission Effect. Two images are arbitrarily selected from the CIFAR database, and three methods are used to visually communicate them. The original image is shown in Figure 3, and the image processing result is shown in Figure 4.

By analyzing Figures 3 and 4 , it can be seen that the visual effect obtained by using the method in this article is good, which not only retains the image detail information, but also does not have the problem of image blur. However, when using traditional methods to process the image, the image has some fuzziness, edge fuzziness, and poor visual communication effect. Therefore, the image processing effect of this method is better.
4.2.2. Image Visual Communication Effect under the Influence of Noise. An arbitrary image is selected as the input in the experimental data set, and the peak signal-to-noise ratio PSNR is used as the objective criterion of evaluation. Set the noise probability to $0.3,0.6$, and 0.9 , respectively, and compare the three methods under different conditions. The experimental results are shown in Figure 5.

It can be seen from the curve in Figure 5 that the three methods are not suitable for the case of strong noise. The higher the noise probability, the lower the peak signal-tonoise ratio. The comparison shows that under different noise probabilities, the peak signal-to-noise ratio of this method is higher than that of the traditional method, and it is obviously better than the traditional method in the whole noise probability range. It shows that in the presence of noise, the traditional methods cannot remove the bright and dark points caused by noise from the image, but this method has a good effect on noise suppression and solves this problem, and the subjective visual effect of the image is greatly improved. In the experiment, it is found that the image visual effect is the best when the noise probability is 0.3 . Therefore, this method has good antinoise performance and good visual communication effect.

4.2.3. The Efficiency of Image Visual Transmission. In addition to the visual communication effect of the image itself, the image processing efficiency is also an important index to measure the method. Due to the rapid improvement of the number of images, the rapid visual communication processing of a large number of images is a key problem for 


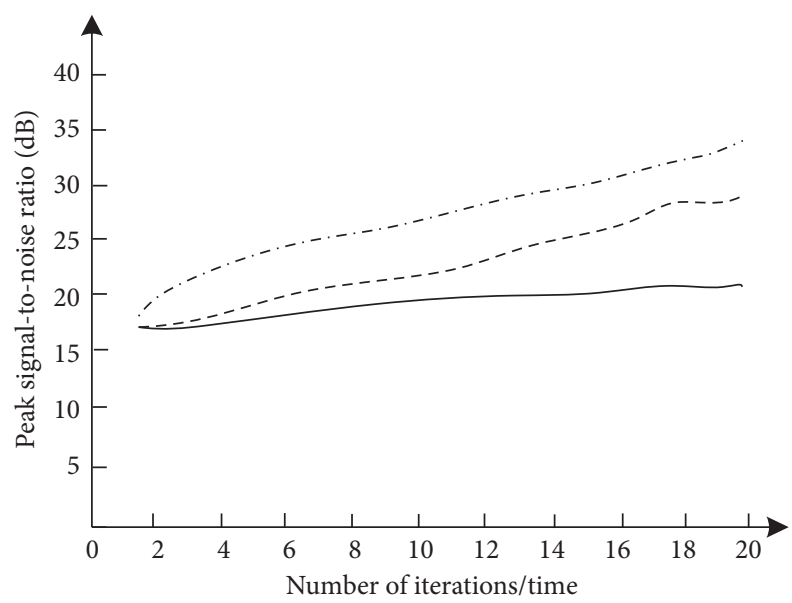

Visual communication method based on wavelet change

_. - Visual communication method based on user experience effect

.... Method of this article

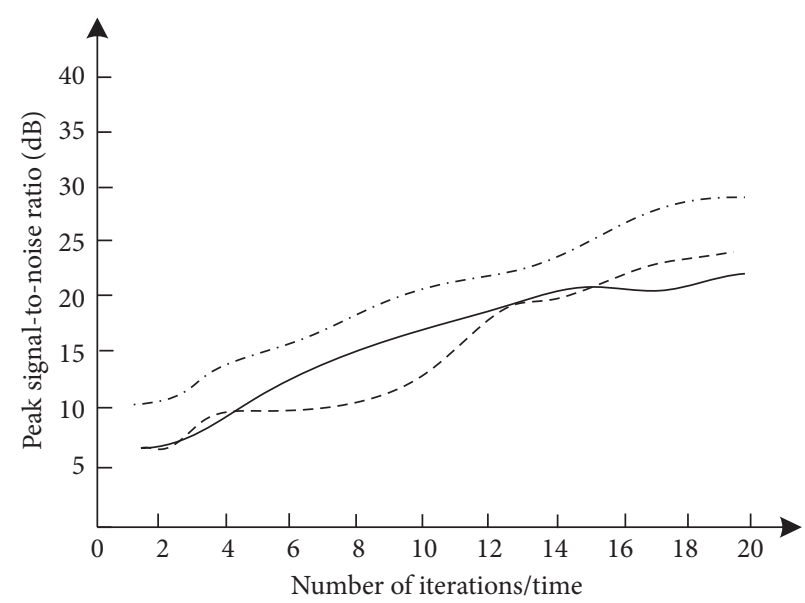

Visual communication method based on wavelet change

- _ - Visual communication method based on user experience effect

... Method of this article

(a)

(b)

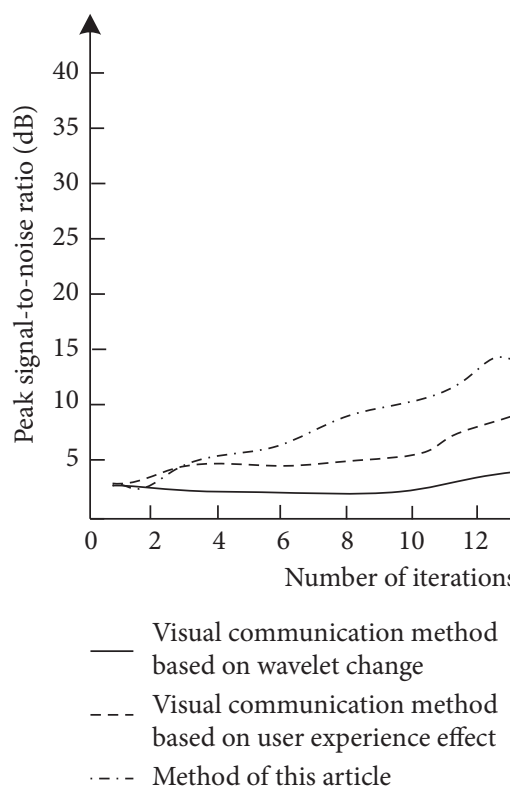

(c)

FIgURE 5: Comparison of antinoise performance. (a) Noise probability 0.3. (b) Noise probability 0.6. (c) Noise probability 0.9.

studies at present. Therefore, taking the image visual communication efficiency as the experimental index, the different methods are compared, and the results are shown in Figure 6.

According to the analysis of Figure 6, with the increase in the number of images, the visual communication efficiency of different methods shows a downward trend, indicating that the visual communication efficiency of images is affected by the number of images. Comparing the visual communication efficiency of different methods, it can be seen that the visual communication efficiency of this method is always higher than the two traditional methods, the highest visual communication efficiency reaches $84 \%$, and the downward trend is slower than the traditional methods. The visual communication efficiency of the plane visual communication effect optimization method based on wavelet change and the plane visual communication design method based on user experience effect is not only significantly lower than that of this method, but also decreases significantly with the increase of the number of images, indicating that the two traditional methods are not suitable for a large number of image processing and have certain limitations.

The artistic visual image stereo communication method is used to perceive the technical action posture data at the gymnastics scene, and the visual reconstruction analysis 


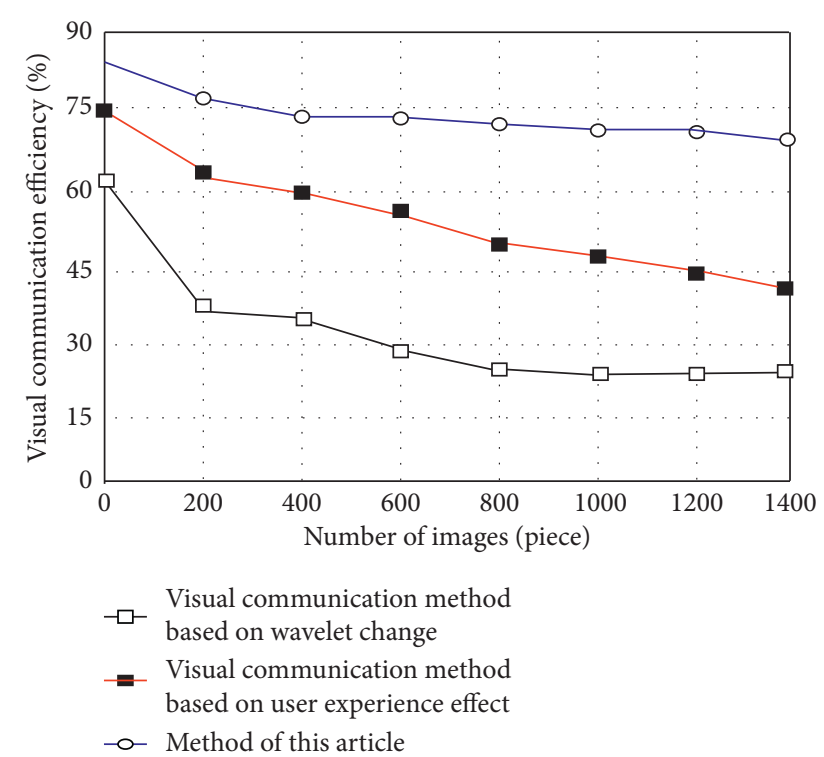

FIGURE 6: Comparison of image visual communication efficiency.

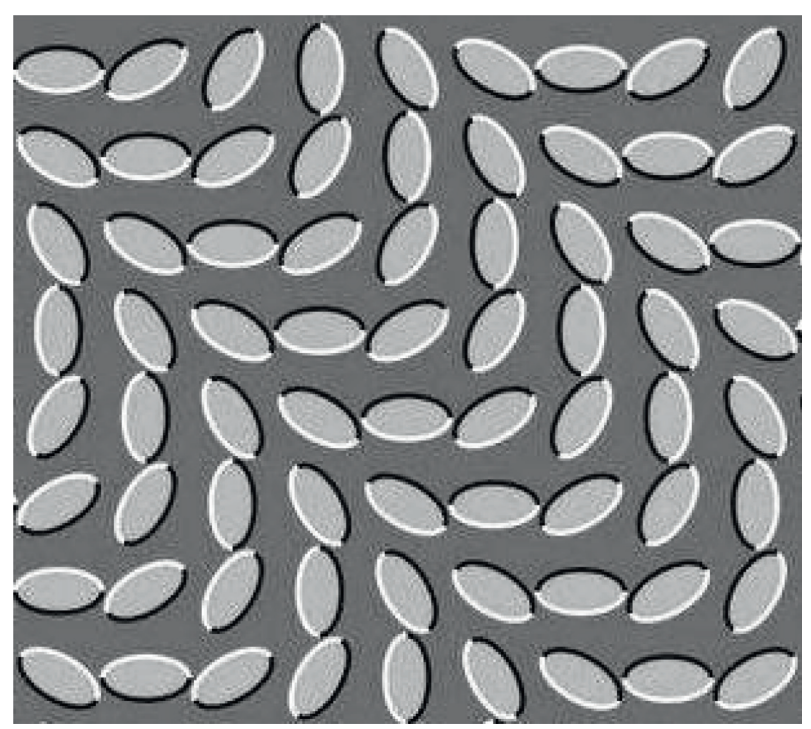

FIgURE 7: Visual reconstruction of art image feature data.

results of artistic image feature data are obtained, as shown in Figure 7.

It can be seen from the results in Figure 7 that through the method designed in this article, the visual reconstruction effect of art image feature data is good, the visual effect of art image can be displayed accurately, and the performance is stable. It provides visual support for the analysis of visual reconstruction of art image feature data, and the performance of the designed method is superior.

Through the above comparative analysis, it can be seen that the image definition, visual communication efficiency, and denoising effect of this method are higher than the traditional plane visual communication effect optimization method based on wavelet change and the plane visual communication design method based on user experience effect. This method can better grasp the basic information of image data and improve the visual communication efficiency, and it has better research value to enhance the performance of image information protection and improve the clarity of image transmission.

\section{Conclusion}

In order to study an effective image processing method, in order to improve the image communication effect, improve the image communication efficiency, and reduce the influence of noise factors on the image, this article proposes a three-dimensional communication method of artistic visual image based on CAD platform. The simulation results show that compared with the traditional methods, the image processing effect of this method is good, which can improve the image transmission effect to a certain extent and improve the clarity and integrity of the image. The performance of the designed method is superior. In the experiment, it is found that when the noise probability is 0.3 , the image visual effect is the best, satisfactory effect can be obtained, and the antinoise performance is good. The visual communication efficiency is always higher than the two traditional methods, and its highest visual communication efficiency reaches $84 \%$, and the downward trend is more slow.

\section{Data Availability}

The raw data supporting the conclusions of this article will be made available by the authors, without undue reservation.

\section{Conflicts of Interest}

The authors declare that they have no conflicts of interest regarding this work.

\section{Acknowledgments}

This work was supported by the Shandong Provincial Key Subject of Art and Science of 2020 , Study on Wetland Cultural Creative Products in Yellow River Delta (QN202008224).

\section{References}

[1] C. Yu, "Retracted article: climate environment of coastline and urban visual communication art design from the perspective of GIS," Arabian Journal of Geosciences, vol. 14, no. 4, p. 310, 2021.

[2] T. Li, Y. Wang, R. Hong, M. Wang, and X. Wu, "pDisVPL: probabilistic discriminative visual Part Learning for image classification," IEEE MultiMedia, vol. 25, no. 4, pp. 34-45, 2019.

[3] Z. Tang, H. Zhang, C. M. Pun, M. Yu, C. Yu, and X. Zhang, "Robust image hashing with visual attention model and invariant moments," IET Image Processing, vol. 14, no. 5, pp. 901-908, 2020.

[4] Z. Hao, X. Wang, and S. Zheng, "Recognition of basketball players' action detection based on visual image and Harris corner extraction algorithm," Journal of Intelligent and Fuzzy Systems, vol. 40, no. 1, pp. 1-11, 2020. 
[5] K. Kyamakya, A. Haj Mosa, F. A. Machot, and J. C. Chedjou, "Document-image related visual sensors and machine learning techniques," Sensors, vol. 21, no. 17, p. 5849, 2021.

[6] D. D. Rodriguez, C. Goulart, C. Valadão, D. Funayama, and T. Bastos, "Visual and thermal image processing for facial specific landmark detection to infer emotions in a child-robot interaction," Sensors, vol. 19, no. 13, p. 2844, 2019.

[7] J. Yin and J. H. Yang, "Virtual reconstruction method of regional 3D image based on visual transmission effect," Complexity, vol. 2021, Article ID 5616826, 12 pages, 2021.

[8] A. Kosovicheva, K. Sridhar, and P. J. Bex, "Image predictors of visual localization in natural scenes," Journal of Vision, vol. 20, no. 11, p. 183, 2020.

[9] M. M. Liu, "Optimization of plane visual communication effect simulation based on graphic beautification technology," Computer Simulation, vol. 36, no. 9, pp. 426-429, 2019.

[10] C. Li, "Research on graphic visual communication design based on user experience effect," Modern Electronics Technique, vol. 43, no. 11, pp. 111-114, 2020.

[11] X. Q. Zhu, "Innovative design of graphic image visual communication system for animated character in virtual reality environment," Modern Electronics Technique, vol. 561, no. 10, pp. 178-180, 2020.

[12] J.-J. Liaw, C.-P. Lu, Y.-F. Huang, Y.-H. Liao, and S.-C. Huang, "Improving census transform by high-pass with haar wavelet transform and edge detection," Sensors, vol. 20, no. 9, p. 2537, 2020.

[13] X. Dong, G. Li, Y. Jia, and K. Xu, "Multiscale feature extraction from the perspective of graph for hob fault diagnosis using spectral graph wavelet transform combined with improved random forest," Measurement, vol. 176, no. 2, Article ID 109178, 2021.

[14] F. Muoz-Muoz and A. Rodrigo-Mor, "Partial discharges and noise discrimination using magnetic antennas, the cross wavelet transform and support vector machines," Sensors, vol. 20 , no. 11 , p. $3180,2020$.

[15] A. Rezapour, A. Ortega, and M. Sahimi, "Upscaling of geological models of oil reservoirs with unstructured grids using lifting-based graph wavelet transforms," Transport in Porous Media, vol. 127, no. 3, pp. 661-684, 2019.

[16] J. Pushparaj and M. Malarvel, "Panchromatic image denoising by a log-normal-distribution-based anisotropic diffusion model," Journal of Applied Remote Sensing, vol. 13, no. 1, p. 1, 2019.

[17] S. Seo, "Image denoising and refinement based on an iteratively reweighted least squares filter," KSCE journal of civil engineering, vol. 24, no. 3, pp. 943-953, 2020.

[18] Y. Wang, D. Chang, and Y. Zhao, "A new blind image denoising method based on asymmetric generative adversarial network," IET Image Processing, vol. 15, no. 6, pp. 1260-1272, 2021.

[19] M. H. Rahman, C. Schimpf, C. Xie, and Z. Sha, "A CAD-based research platform for data-driven design thinking studies," Journal of Mechanical Design, vol. 141, no. 12, p. 1, 2019.

[20] C. Dasgupta, A. J. Magana, and C. Vieira, "Investigating the affordances of a CAD enabled learning environment for promoting integrated STEM learning," Computers \& Education, vol. 129, no. 2, pp. 122-142, 2019.

[21] A. Jhld, "Fabricating a crown under an existing removable partial denture with impression scanning and CAD-CAM technology," The Journal of Prosthetic Dentistry, vol. 124, no. 2, pp. 148-152, 2020.

[22] Z. Chen, Y. Wang, and Z. Song, "Classification of motor imagery electroencephalography signals based on image processing method," Sensors, vol. 21, no. 14, p. 4646, 2021.
[23] H. Zhang and J. Yao, "Automatic focusing method of microscopes based on image processing," Mathematical Problems in Engineering, vol. 2021, Article ID 8243072, 9 pages, 2021.

[24] W. Zhang, X. Li, Q. Song, and W. Lu, "A face detection method based on image processing and improved adaptive boosting algorithm," Traitement du Signal, vol. 37, no. 3, pp. 395-403, 2020.

[25] F. Han, J. Yao, H. Zhu, and C. Wang, "Underwater image processing and object detection based on deep CNN method," Journal of Sensors, vol. 2020, Article ID 6707328, 20 pages, 2020.

[26] N. H. Hai, H. L. Minh, D. N. H. Thanh, N. V. Son, and S. Prasath, "An adaptive image inpainting method based on the weighted mean," Informatica, vol. 43, no. 4, pp. 507-513, 2019.

[27] B. Nga, B. Sta, B. Mkea, M. N. Mab, and B. Nla, "Image postprocessing adoption trends in clinical medical imaging," Journal of the American College of Radiology, vol. 16, no. 7, pp. 945-951, 2019.

[28] B. Hadhami, R. Slama, R. Gaha, and A. Benamara, "Proposal of new eco-manufacturing feature interaction-based methodology in CAD phase," International Journal of Advanced Manufacturing Technology, vol. 106, no. 3, pp. 1057-1068, 2020.

[29] J. Qin, J. Chen, X. Xiang, Y. Tan, W. Ma, and J. Wang, “A privacy-preserving image retrieval method based on deep learning and adaptive weighted fusion," Journal of Real-Time Image Processing, vol. 17, no. 1, pp. 161-173, 2020.

[30] S. Vaez and Z. Minaei, "Pulse extraction of pulselike ground motions based on particle swarm optimization algorithm," Scientia Iranica, vol. 27, no. 1, pp. 134-158, 2020.

[31] J. Zhang, Z. Sun, N. Qiu, Y. Zhang, and F. Li, "3-D effetive elastic thickness inversion of subduction zone based on particle swarm optimization algorithm," Chinese Journal of Geophysics- Chinese Edition, vol. 62, no. 12, pp. 4738-4749, 2019.

[32] X. N. Bui, P. Jaroonpattanapong, H. Nguyen, Q. H. Tran, and N. Q. Long, "A novel hybrid model for predicting blast-induced ground vibration based on k-nearest neighbors and particle swarm optimization," Scientific Reports, vol. 9, no. 1, pp. 1-14, 2019.

[33] W. F. Fihri, H. E. Ghazi, and B. Majd, "A multi-objective particle swarm optimization based algorithm for primary user emulation attack detection," Wireless Personal Communications, vol. 117, no. 8, pp. 1-20, 2021.

[34] K. Malik and F. Kim, "Optimal travel route recommendation mechanism based on neural networks and particle swarm optimization for efficient tourism using tourist vehicular data," Sustainability, vol. 11, no. 12, p. 3357, 2019.

[35] V. Santucci, M. Baioletti, and A. Milani, "Tackling permutation-based optimization problems with an algebraic particle swarm optimization algorithm," Fundamenta Informaticae, vol. 167, no. 1-2, pp. 133-158, 2019.

[36] B. Xiao and S.-C. Kang, "Development of an image data set of construction machines for deep learning object detection," Journal of Computing in Civil Engineering, vol. 35, no. 2, Article ID 05020005, 2021. 